\title{
Health literacy and preparedness of health workers in the private health sector towards the COVID-19 pandemic: Need for integration into the nationwide response
}

\author{
Ekaete Tobin, ${ }^{1}$ Vivian Ajekweneh, ${ }^{2}$ Andrew Obi, ${ }^{3}$ Eshan Henshaw ${ }^{4}$ \\ ${ }^{1}$ Institute of Lassa Fever Research and Control, Irrua Specialist Teaching Hospital, Irrua, Edo State, \\ Nigeria; ${ }^{2}$ Department of Community Medicine, Irrua Specialist Teaching Hospital, Irrua, Edo State, \\ Nigeria; ${ }^{3}$ Department of Public Health and Community Medicine, University of Benin Teaching Hospital, \\ Benin City, Edo State; ${ }^{4}$ Department of Internal Medicine, University of Calabar, Calabar, Cross River State, \\ Nigeria
}

\begin{abstract}
The private health sector has the potential to participate in the COVID-19 pandemic response. The study aimed to assess the
\end{abstract}

\author{
Correspondence: Ekaete Tobin. Institute of Lassa Fever Research and \\ Control, Irrua Specialist Teaching Hospital, Km 87 Benin-Auchi Road, \\ Irrua, Edo State, Nigeria. \\ Tel.:+2348155368412. \\ E-mail: ekaete.tobin@gmail.com
}

Key words: General Practice; COVID-19 pandemic; private sector.

Acknowledgements: The authors are grateful to all the health workers who voluntarily participated in the survey, and to Miss Ameh Anabue for proofreading the manuscript.

Contributions: ET and VA developed the concept note; ET, VA and AO coordinated data collection. ET analysed the data using the data analysis plan agreed upon by all authors. The draft manuscript was written by ET, VA, EH and all authors read and approved the final manuscript.

Conflict of interest: The authors declare no conflict of interest.

Availability of data and materials: All data generated or analyzed during this study are available upon request to the authors.

Ethics approval and consent to participate: The Ethics Committee of Irrua Specialist Teaching Hospital approved this study (ISTH/HREC/20201019/127). The study conforms with the Helsinki Declaration of 1964, as revised in 2013, concerning human and animal rights.

Informed consent: Written informed consent was obtained from all respondents for anonymized personal information to be published in this article.

Received for publication: 10 March 2021.

Revision received: 22 March 2021.

Accepted for publication: 15 Aptil 2021.

This work is licensed under a Creative Commons Attribution NonCommercial 4.0 License (CC BY-NC 4.0).

(C) Copyright: the Author(s) 2020

Licensee PAGEPress, Italy

Annals of African Medical Research 2020; 3:145

doi:10.4081/aamr.2020.145 health literacy, perceptions, practices, willingness to participate and opportunities for engagement of the private health sector in the COVID-19 response. A cross-sectional survey was carried out among health workers in private health facilities in Edo Central and Edo North Senatorial districts of Edo state between May and June 2020. Data were collected using pre-tested questionnaires and analysis carried out using Statistical Package for Social Science (SPSS). Chi-square test of significance and logistic regression were applied at $5 \%$ cut off. A total of 153 health workers participated giving a response rate of $75.0 \%$. Eighty-eight $(57.5 \%)$ respondents had good knowledge of COVID-19 and $80(52.3 \%)$ held negative perceptions towards COVID-19. Ninety-five $(62.1 \%)$ respondents believed private health facilities had a role to play in the response particularly in the area of suspected case screening (85.4\%). Thirty-one (20.3\%) respondents indicated their willingness to participate in the COVID-19 response if their facilities were invited to. Sixty-one (39.9\%) and 92 (60.1\%) respondents respectively held poor and good practices towards COVID19 prevention, with practice significantly associated with educational level $\left(\chi^{2}=14.10, \mathrm{P}<0.01\right)$, profession $\left(\chi^{2}=15.28, \mathrm{P}=0.01\right)$. and previous training in infection prevention and control (IPC) $\left(\chi^{2}\right.$ $=18.16, \mathrm{P}<0.01)$. The resources available from the private sector to support the response can be harnessed through engagements with medical directors and health workers in the sector to identify areas of collaboration, address identified gaps in knowledge, improve perception and participation.

\section{Introduction}

Coronavirus Disease 2019 also known as COVID-19 is a potentially severe acute respiratory infection caused by coronavirus 2 (SARS-CoV-2). ${ }^{1}$ Probably of zoonotic origin, human-tohuman transmission, which is responsible for the pandemic spread of the disease, occurs through inhalation of respiratory droplets during sneezing, coughing or talking from an infected individual. ${ }^{2}$ The elderly and persons with pre-existing illnesses (hypertension, Diabetes Mellitus, cardiac disease, chronic lung disease, cancer, or Asthma) have a higher risk for severe disease and mortality. ${ }^{3}$

Healthcare workers are at risk of contracting the SARS-CoV-2 virus through their interactions with patients in the healthcare setting, irrespective of their job description. ${ }^{4}$ In a developing country like Nigeria, patronage of private practitioner hospitals is high ${ }^{5}$ and the likelihood of healthcare workers in those establishments coming in contact with persons who may potentially be infected with the virus is higher than the general population. ${ }^{6}$ Hence it is of utmost importance that all healthcare workers have good knowl- 
edge of this novel disease, the right perception and adopt safe preventive practices. Moreover, the speed with which the disease became a pandemic, and the limited information about the disease has led to an abundance of false information over the internet and social media platforms, thus creating negative perceptions and fear among health workers. ${ }^{7}$ Fear drives panic and stigma, and both lead to the hesitancy of health workers to render services and in many instances, neglect of suspected cases, even in situations where initial medical attention should be offered to these persons while they await the results of COVID-19 laboratory tests. ${ }^{7,8}$

The scale of the pandemic has also overwhelmed the government health system in many countries and created shortages of competent trained frontline healthcare workers and essential commodities, forcing governments to look towards the private formal health sector for surge capacity. The need to ensure that the private health sector COVID-19 related activities are not only aligned with national response efforts but also delivered in such a manner that is safe for patients and health workers becomes paramount. Countries with a pre-existing mechanism of private sector engagement were able to do this more efficiently than those without established mechanisms of engagement. Both can benefit from practical, hands-on, tailored guidance to effectively work with the private sector during the COVID-19 crisis. Private health providers too are looking for ways to contribute but are not well-positioned to work effectively with the government. ${ }^{9}$

The study sought to assess the knowledge and perceptions of healthcare workers in private practitioner's health facilities have towards COVID-19. This will assist in identifying the knowledge gap among healthcare staffs, which will inform existing policy modification that may mitigate the spread of COVID-19 among healthcare workers and identify potential areas of collaboration.

\section{Materials and Methods}

\section{Study area and population}

The study was carried out in Edo state, located in the SouthSouth geopolitical zone of Nigeria. The projected population of the state as in 2019 was 4,592,961, spread across an area of about 19,743 sq. $\mathrm{km}$. The boundaries of the state are Kogi State to the North-East, Delta state to the South-East, Anambra State to the East, and Ondo State to the West and the North-West. Edo state is divided into 3 senatorial districts: Edo North, Edo Central and Edo south, each with 5 local government areas (LGA). The health system of the state comprises the formal and informal sector, with the formal sector including privately owned and public health facilities. There are about 345 registered private hospitals in the state. ${ }^{10}$

The study population were staff of private hospitals in the study area. A large number of these facilities operate as sole proprietorship or joint ventures with nurse midwives and auxiliaries, followed by doctors as the predominant work categories. ${ }^{11}$ Other categories of staff found in private hospitals include laboratory technicians, pharmacists, cleaners and gate keepers.

\section{Study design and sample size}

The cross-sectional survey was carried out between May 2020 and June 2020 with a sample size of 204 calculated using the formula for a prevalence study in a populations less than 10,000: $\mathrm{nf}=$ $\mathrm{n}_{\mathrm{o}} /\left[1+\mathrm{n}_{\mathrm{o}} / \mathrm{N}\right]$. with the assumption of a prevalence value of $78.9 \%,{ }^{12}$ a $5 \%$ margin of error, confidence interval of $95 \%$ and $10 \%$ non-response rate.

\section{Selection criteria}

All employees of private practitioner health facilities, regardless of the time of employment, who were present at the time of conduct of the study and were willing to consent were eligible to participate in the study.

\section{Sampling technique}

The study was carried out in Edo Central and Edo North senatorial districts of Edo State. A list of registered General medical practice facilities in the selected senatorial districts was obtained from the State health management board and stratified by local government area. A rapid assessment provided the total number of healthcare workers in the listed facilities. Population proportionate to size sampling was used to determine the required number of health workers for each senatorial district. Using the assumption of 6 health workers in a facility, ${ }^{11}$ population proportionate to size sampling was used to determine the number of health facilities to be selected in each LGA, and thereafter simple random sampling applied to select the health facilities. In all selected facilities, eligible health workers were invited to participate.

\section{Data collection tools}

Data were collected using structured interviewer-administered questionnaires developed after extensive research on the topic. The questionnaire was divided into four parts. The first part dealt with sociodemographic characteristics. Part 2 contained 24 questions on basic knowledge of COVID-19. A correct response was scored one point, while an incorrect answer or 'I do not know' response was scored 0, with the maximum score as 24 . Knowledge of COVID19 was graded as good if he/she scored $>75 \%$ of total scores, average if between $50-75 \%$ and poor if $<50 \%$.

Part 3 included 9 questions on perceptions toward COVID-19, with Likert-style responses coded as strongly agree/ agree $=2$, Uncertain $=0$ and disagree/Strongly disagree $=1$. Perception of confidence in using personal protective equipment required a choice from 'confident' scored as 1, 'not confident' or 'not sure', both scored as 0 with total score for perception as 19 . Perception was graded as healthy perception for a score $\geq 50 \%$ of total, and unhealthy if $<50 \%$.

Part 4 contained 8 questions on preventive practices adopted by respondents with responses as 'always', 'sometimes', 'rarely', and 'never'. A score of 1 was assigned to good behaviour which was always or sometimes practised, and 0 if rarely or never practised. The total score for practice was 14, with good practice assigned to a grade $\geq 50 \%$ of the total, and poor if $<50 \%$. Willingness to participate in the COVID-19 response was assessed by the question 'Would you be willing to participate in the response if your facility was designated a centre?', with response options as 'Yes' or 'No'. Factors that may influence willingness to participate and potential areas of involvement of the private health sector were assessed.

Face and content validity of the study questionnaire was carried out to ascertain the validity of the data collection tool. The reliability of the tool was assessed using the test-retest method with the tool administered to 2 groups of 5 community residents twice in a space of 2 weeks. Cronbach's alpha coefficient was calculated as 0.72 .

\section{Study variables}

Age, sex, profession, educational level, marital status, years of experience, training in COVID-19, training in IPC and factors associated with willingness to participate were exposure variables. 
Knowledge of COVID-19, perceptions towards COVID-19 and willingness to participate in the response were outcome measures.

\section{Data analysis}

Data analysis was done using SPSS version 20 (IBM Corp., USA). Descriptive data on sociodemographic characteristics were presented in frequency distribution tables. Means and standard deviation or median were calculated for continuous variables. The associations between exposure and outcome variables were tested using the Chi-square test of association with the level of statistical significance set as $\mathrm{p}<0.05$.

\section{Ethical considerations}

Ethical approval was received from the Irrua Specialist Teaching Hospital Ethics Committee. Informed consent was obtained from study participants who were told participation was voluntary and that they could withdraw at any time. Anonymized data was used for analysis and interpretation and data collection tools kept confidential. All participants provided consent after a detailed instruction describing the main purposes and approximate duration of the study was provided to them. The interviewers wore protective face masks. Reasonable physical distance was kept between the involved individuals during data collection. The potential risk of exposure to the virus was minimal at the time of the study. Following data collection, research assistants were observed for 14 days.

\section{Results}

One hundred and fifty-three health workers participated giving a response rate of $75.0 \%$. The mean age of respondents was 29.6 (9.6) years. Table 1 shows the demographic profile of respondents.

All respondents were aware of COVID-19. Twenty-nine
$(19.0 \%)$ respondents had been trained on COVID-19, $75(49.0 \%)$ had been trained on basic IPC, $25(16.3 \%)$ had been trained on both. Correct responses to the knowledge questions are presented

Table 1. Sociodemographic characteristics of respondents $(n=153)$.

\begin{tabular}{lc} 
Variable & Frequency (\%) \\
Age group (years) & \\
$\leq 24$ & $52(34.0)$ \\
$25-34$ & $70(45.8)$ \\
$\geq 35$ & $31(20.3)$ \\
Sex & \\
Male & $39(26.2)$ \\
Female & $110(73.8)$ \\
\hline Marital Status & \\
Single & $78(52.3)$ \\
Married & $66(44.3)$ \\
Divorced/widowed/separated & $5(3.4)$ \\
Educational level & \\
Primary & $4(2.7)$ \\
Secondary & $60(40.3)$ \\
Tertiary & $85(57.0)$ \\
\hline Profession & \\
Nurse & $99(64.7)$ \\
Doctor & $7(4.6)$ \\
Laboratory technician/scientist & $14(9.2)$ \\
Pharmacist/technician & $5(3.3)$ \\
Hygienist & $8(5.2)$ \\
Others* & $20(13.1)$ \\
Years in practice (years) & \\
$\leq 4$ & $78(51.0)$ \\
$5-9$ & $43(28.1)$ \\
$\geq 10$ & $32(20.9)$ \\
\hline
\end{tabular}

$*^{*}$ thers $=$ clerical staff, medical records, security men

Table 2. Responses to knowledge questions among respondents $(\mathrm{n}=153)$.

\begin{tabular}{lc}
\hline Knowledge question & Correct responses n (\%) \\
Agent responsible for COVID-19 & $140(91.5)$ \\
Symptoms of COVID-19 & $144(94.1)$ \\
Fever & $138(90.2)$ \\
Dry cough & $140(91.5)$ \\
Difficulty in breathing & $109(71.2)$ \\
Sore throat & $46(30.1)$ \\
Myalgia & $54(35.3)$ \\
Fatigue & $63(41.2)$ \\
Chest pain & $117(76.5)$ \\
\hline Methods of spread & $146(95.4)$ \\
Through contaminated medical devices & $111(72.5)$ \\
Droplets released during coughing and sneezing & $150(98.0)$ \\
Through infected animal vectors & $110(71.9)$ \\
Through contaminated food & $140(91.5)$ \\
Through contact with blood or body fluid from an infected person & \\
Incubation period & $111(72.5)$ \\
Prevention & $85(55.6)$ \\
Avoid going to crowded places & $150(98.0)$ \\
Maintaining a safe distance from sick persons & $140(91.5)$ \\
Hand washing & $82(53.6)$ \\
Cough etiquette & $143(93.5)$ \\
Isolation of close contacts of sick persons & $127(83.0)$ \\
Early treatment improves outcome for infected persons & $116(75.8)$ \\
Some persons may be asymptomatic & \\
Transmission within hospitals is common &
\end{tabular}


in Table 2.

Eighty-eight $(57.5 \%)$ respondents had good knowledge of COVID -19, 65 (42.5\%) had average knowledge. No respondent had poor knowledge. Good knowledge was significantly associated with being a doctor or laboratory personnel $\left(\chi^{2}=23.43, \mathrm{P}<\right.$ $0.01)$. Educational level $\left(\chi^{2}=11.35, \mathrm{P}<0.01\right)$ and training in IPC $\left(\chi^{2}=6.61, \mathrm{P}=0.01\right)$ were also significantly associated with knowledge (Table 3).

Sixty-nine (45.1\%) respondents believed COVID-19 was developed as a biological weapon, $91(59.5 \%)$ that it was related to $5 \mathrm{G}$, and $65(42.5 \%)$ were not in favour of staying at home to reduce exposure to the virus. Perceptions of COVID-19 are presented in Table 3. Eighty (52.3\%) respondents held negative perceptions towards COVID-19, 73 (47.7\%) held positive perceptions. Perception was significantly associated with previous training in COVID-19 $\left(\chi^{2}=6.48, \mathrm{P}=0.01\right)$, and training in IPC $\left(\chi^{2}=\right.$ $10.94, \mathrm{P}<0.01$, Table 4$)$. Perception was however not significantly associated with knowledge $\left(\chi^{2}=0.66, P=0.42\right)$. Sixty-one $(39.9 \%)$ held poor practices towards COVID-19 prevention, $92(60.1 \%)$ had good practices, with practice significantly associated with educational level $\left(\chi^{2}=14.10, \mathrm{P}<0.01\right)$, profession $\left(\chi^{2}=15.28, \mathrm{P}=\right.$ $0.01)$. and previous training in IPC $\left(\chi^{2}=18.16, \mathrm{P}<0.01\right)$ Table 4.

Practice was not significantly associated with knowledge $\left(\chi^{2}=\right.$ $1.06, \mathrm{P}=0.30)$, but was significantly associated with perception, as $67(91.8 \%)$ of respondents with positive perceptions had good practice, compared with $25(31.2 \%)$ respondents with negative perceptions towards COVID-19. Similarly, 6 (8.2\%) respondents with positive perceptions had poor practice compared with 55 $(68.8 \%)$ of persons with negative perceptions $\left(\chi^{2}=58.34, \mathrm{P}<\right.$ $0.001)$.

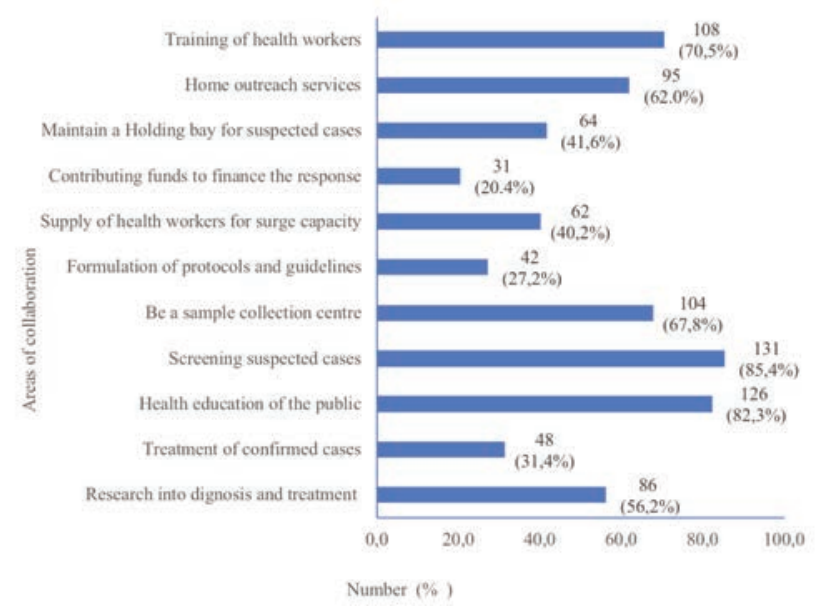

Figure 1. Areas of possible involvement of the private formal health sector in the COVID-19 response (multiple response).
Ninety-five (62.1\%) respondents believed private health facilities had some role to play in the COVID-19 response. Areas where possible involvement in the response were identified, are shown in Figure 1.

Only $31(20.3 \%)$ respondents indicated their willingness to participate in the COVID-19 response if their facility were to be approached by the State government response team (Figure 2).

Twenty-eight $(90.3 \%)$ of those who expressed willingness did so as they felt duty-bound to save lives by reason of their training $\left(\chi^{2}=11.65, \mathrm{P}<0.001\right), 27(87.1 \%)$ for the love of their fellow citizens $\left(\chi^{2}=21.03, \mathrm{P}<0.001\right), 23(74.2 \%)$ opined it an opportunity for career advancement $\left(\chi^{2}=10.92, \mathrm{P}<0.001\right)$, and $17(54.8 \%)$ as a means to gain skills and experience $\left(\chi^{2}=7.01, \mathrm{P}<0.001\right)$. Coercion and desire to follow colleagues were not significant reasons for willingness to participate $\left(\chi^{2}=0.06, \mathrm{P}=0.81\right.$ and $\chi^{2}=$ $1.75, \mathrm{P}=0.19$ respectively).

The most common reason given by respondents who would not

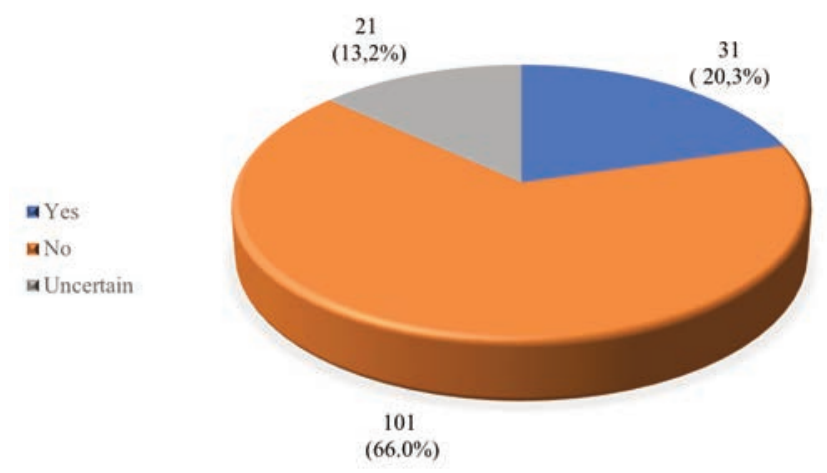

Figure 2. Respondents' willingness to participate in the COVID19 response in the state $(n=153)$.

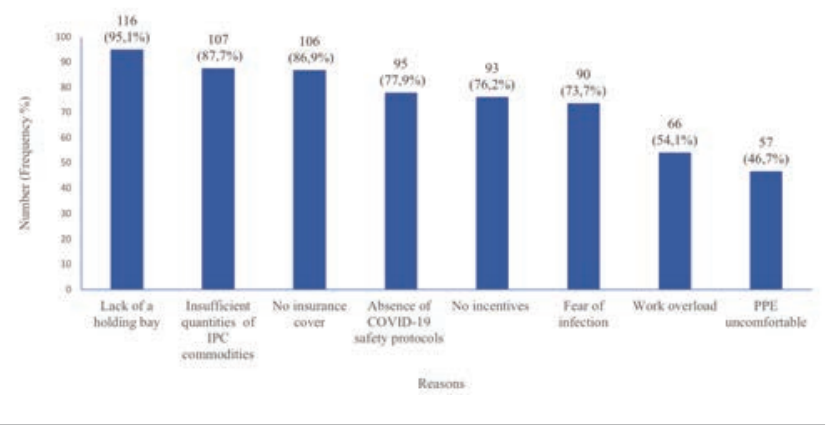

Figure 3. Factors that may deter respondents from participating in the COVID-19 response (multiple response).

Table 3. Attitudinal responses from participants $(n=153)$.

\begin{tabular}{lccc} 
Variable & Strongly agree/Agree & Uncertain & Strongly disagree/disagree \\
I think COVID-19 is related to 5G & $28(18.3)$ & $63(41.2)$ & $62(40.5)$ \\
COVID-19 was developed as a biological weapon & $69(45.1)$ & $52(34.0)$ & $32(21.0)$ \\
\hline I believe COVID-19 is a deadly disease & $73(47.7)$ & $38(24.8)$ & $42(27.4)$ \\
I feel herbs are effective against COVID-19 infection & $6(3.9)$ & $43(28.1)$ & $104(68.0)$ \\
\hline Warm water can reduce the risk of getting COVID-19 & $43(28.1)$ & $44(28.8)$ & $66(43.1)$ \\
I believe antibiotics can treat COVID-19 & $25(16.3)$ & $46(30.1)$ & $73(53.6)$ \\
\hline I do not believe staying at home can prevent contracting COVID-19 & $65(42.5)$ & $74(48.2)$ & $74)$ \\
\hline
\end{tabular}




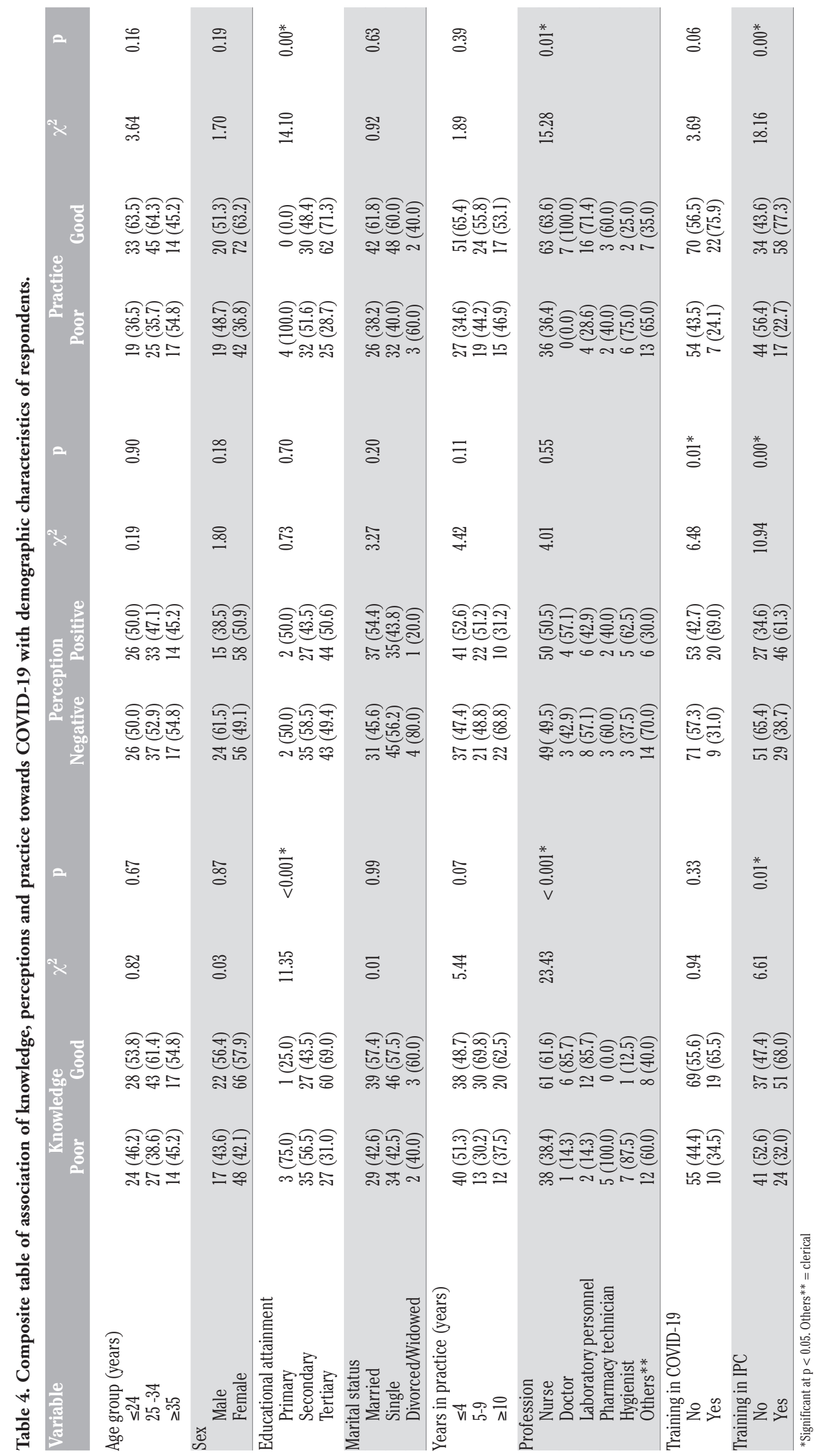


be willing to participate was the lack of a holding bay in their facility, 116 (95.1\%). Other reasons given are shown in Figure 3.

\section{Discussion}

The study showed health workers in private medical practice had a reasonable degree of health literacy of COVID-19, about 1 out of 2 held negative perceptions about the disease, and two-thirds reported good practices in terms of following COVID-19 precautionary measures. The high proportion with considerably good knowledge, even though only a small proportion had received any formal training on COVID-19, is comparable to a study carried out among general practitioners in Australia. ${ }^{13}$ This points to a desire for health workers in the private sector to be informed about the disease. Indeed health workers have been found to on their own seek information on COVID-19 from social media sources and government websites. ${ }^{14-16}$ Respondents were conversant with fever and the respiratory manifestations of the disease. However, only a few knew other constitutional symptoms commonly associated with the disease such as myalgia and fatigue. Most were ignorant of the need to isolate close contacts and maintain a safe distance from sick persons. These gaps in knowledge may reduce their index of suspicion or ability to provide appropriate counsel to contacts of suspected or confirmed cases. These gaps in knowledge can be addressed through increasing the number of health workers who are trained and ensuring that training modules are sufficiently robust in content. The finding of a statistically significant association between having received training on COVID-19 and practice of preventive behaviours has been observed in another study. ${ }^{4}$ The greater proportion of clinical-related professions with good knowledge and preventive practices compared to hygienists and nonclinical staff may be due to the leverage provided by their medical background and highlights the need to tailor trainings to fit the comprehension of the target audience, and engage all professional groups in behaviour change strategies to increase uptake of precautionary measures.

Slightly more respondents were found to have negative compared to positive perceptions about COVID-19. This finding is shared by an earlier study ${ }^{17}$ and may from believing misconceptions and misinformation on COVID-19 that are abundant on social media. COVID-19 was regarded as a biological weapon and related to the fifth-generation mobile telecommunication technology, popularly known as $5 \mathrm{G}$, by a considerable number of respondents. A multi-country study found $14 \%, 6.3 \%$ and $8.4 \%$ of respondents drawn from Central Africa, West Africa and East Africa respectively to hold the same view. ${ }^{18}$ In another study in the United Kingdom, $21.3 \%$ and $45.4 \%$ of respondents respectively upheld the theory of COVID-19 related to bioterrorism and 5G network service respectively. ${ }^{19}$ The rapid spread of these misconceptions may have been fuelled by the paucity of knowledge about the virus, the failure of telecommunication companies and the government to provide evidence of $5 \mathrm{G}$ capabilities and the stance taken by influential persons in society. ${ }^{7,20}$ The danger here is that health workers should be custodians of knowledge, so even though in the private sector they are not directly involved in COVID-19 patient care, they should be able to dispel rumours. These misconceptions should be addressed by active engagements between government, key COVID-19 response players and health workers in the private sector.

Attendance at a COVID-19 specific training led to better knowledge of COVID-19 and compliance with preventive prac- tices though not statistically significant. On the other hand, training in infection prevention and control produced significant changes in all domains yet was attended by fewer respondents. A similar finding has previously been documented. ${ }^{4}$ IPC on the other hand, as a measure to address not only COVID-19 but also other sources of unidentified infections, has only gained relevance in recent years. There is a need for more IPC training for private health workers.

The majority of the respondents maintained good preventive practices towards COVID-19, as has been documented in other studies. ${ }^{15,16}$ The finding from the study, that private health workers recognised the potential for private formal health sector involvement in the response is encouraging and such collaboration should be explored by State health ministries through the engagement of the relevant health associations in the private health sector. Indeed the wide geographical spread of private hospitals and the preference for locals to patronize them ${ }^{11,21}$ positions them favourably to deliver a number of the services identified by the respondents, particularly those that do not increase their risk of occupational exposure to COVID-19.

The World health organisation has advised governments to adopt a 'whole-of-government and whole-of-society' approach in their COVID-19 response, bringing in the private forma health sector and civil society groups as they could offer extra capacity for response efforts. ${ }^{22}$ Despite this laudable call, the study showed that only 1 -in- 5 health workers were willing to participate in the response. The lack of IPC commodities, health insurance cover and facility-specific guidelines and protocols have similarly been cited in another study as reasons for low interest in the response among health workers. ${ }^{14}$ On the other hand, the private health sector in some countries has been effectively engaged in teleconsultations, building laboratory diagnostic capacity, case detection and control, public awareness campaigns, and the supply of data needed for COVID-19 surveillance and planning of health services. ${ }^{23-25}$

The study has a few limitations that may have minimal impact on external validity. The study relied on self-report by the health workers, and validation of their responses was not carried out due to the cross-sectional nature of the study design. The study was also carried out in senatorial districts in the state that had lower number of COVID-19 cases and may have yielded different results if the hotspot senatorial districts were also examined.

\section{Conclusions}

The majority of health workers engaged in private hospitals had good knowledge of COVID-19 and good preventive practices, though the number with negative perceptions was slightly above those with positive perceptions. Misconceptions and gaps in knowledge and perception have been identified and should be corrected through targeted training. The majority were in agreement that the private health sector should be engaged in COVID-19 response, however, only a small number expressed the willingness to participate if called upon. The human and material resources available from the private formal health sector to support the response is largely untapped and can be harnessed through engagement of medical directors and health workers in the sector to identify areas of collaboration to improve knowledge, perception and participation. 


\section{References}

1. Unal M, Irez T. COVID 19 disease caused by coronavirus 2 (SARS-CoV-2) (Severe Acute Respiratory Syndrome). Asian J Med Heal 2020;1-11.

2. Bhagavathula AS, Aldhaleei WA, Rahmani J, et al. Knowledge and Perceptions of COVID-19 Among Health Care Workers: Cross-Sectional Study. JMIR Public Heal Surveill 2020;6:e19160

3. Tian S, Hu N, Lou J, et al. Characteristics of COVID-19 infection in Beijing. J Infect 2020;80:401-6.

4. Asemahagn MA. Factors determining the knowledge and prevention practice of healthcare workers towards COVID-19 in Amhara region, Ethiopia: A cross-sectional survey. Trop Med Health 2020;48:72.

5. Adewumi I, Akinyele C. Healthcare delivery system and preventive health: a review of Sojourn of Lagos State. J Res Human Sci 2018;6:46-59.

6. Singh H, Sharma S. Concerns of frontline doctors in India during COVID-19: A cross-sectional survey. Indian J Public Health 2020;64:S237-9.

7. Barua Z, Barua S, Aktar S, et al. Effects of misinformation on COVID-19 individual responses and recommendations for resilience of disastrous consequences of misinformation. Prog Disaster Sci 2020;8:100119.

8. Cuan-Baltazar JY, Muñoz-Perez MJ, Robledo-Vega C, et al. Misinformation of COVID-19 on the internet: Infodemiology study. JMIR Public Heal Surveill 2020;6:e18444.

9. World Health Organization. Supporting private sector engagement during COVID-19. Health Systems Governance Collaborative 2020;1-4.

10. CIUCI Consulting. Healthcare delivery report: Edo State. Accessed 10 October 2020. Available from: https://ciuci.us/wp-content/uploads/2019/08/Edo-HealthcareReport-Final-V3.pdf

11. Johnson D, Woodman B, Baruwa S, et al. A census of private health facilities in six states of Nigeria. Bethesda, MD: Strengthening Health Outcomes through the Private Sector Project, Abt Associates Inc; 2014.

12. Ayinde $\mathrm{O}$, Usman AB, Aduroja $\mathrm{P}$, et al. A cross-sectional study on Oyo State health care workers knowledge, attitude and practice regarding corona virus disease 2019 (COVID-19). Epub ahead of print 27 April 2020. DOI: 10.21203/rs.3.rs23501/v1.

13. Sotomayor-Castillo C, Nahidi S, Li C, et al. General practitioners' knowledge, preparedness, and experiences of managing
COVID-19 in Australia. Infect Dis Health 2021 Feb 18;S2468 0451(21)00004-3. doi: 10.1016/j.idh.2021.01.004. Online ahead of print.

14. Ogolodom MP. Knowledge, Attitudes and Fears of HealthCare Workers towards the Corona Virus Disease (COVID-19) Pandemic in South-South, Nigeria. Heal Sci J;14:002.

15. Saqlain M, Muddasir Munir M. Knowledge, Attitude and Practice among Healthcare Professionals regarding COVID19: A cross-sectional survey from Pakistan. Journal Hosp Infect 2020;105:419-23.

16. Zhang M, Zhou M, Tang F, et al. Knowledge, attitude, and practice regarding COVID-19 among healthcare workers in Henan, China. J Hosp Infect 2020;105:183-7.

17. Kumar H, Singh Khurana M, Singh Charan G, et al. Knowledge and Perception of Health Professionals towards COVID-19. Int J Health Sci Res 2020;10:123-9.

18. Ovenseri-Ogbomo G, Ishaya T, Osuagwu UL, et al. Factors associated with the myth about 5G network during COVID-19 pandemic in sub-Saharan Africa. J Glob Heal Reports 2020;113.

19. Freeman D, Waite F, Rosebrock L, et al. Coronavirus conspiracy beliefs, mistrust, and compliance with government guidelines in England. Psychol Med 2020;1.

20. Ndinojuo B-CE. 5G, Religion, and misconceptions in communication during Covid-19 in Nigeria. J Messenger 2020;12:97.

21. Ofoli JNT, Ashau-Oladipo T, Hati SS, et al. Preventive healthcare uptake in private hospitals in Nigeria: A cross-sectional survey (Nisa premier hospital). BMC Health Serv Res 2020;20:273.

22. WHO. Supporting private sector engagement during COVID19 - WHO's approach. World Health Organisation; 2020. ACCESSED 10 October 2020. Available from: https://www.who.int/publications/i/item/supporting-privatesector-engagement-during-covid-19-who-s-approach

23. Nimako BA, Baiden F, Awoonor-Williams JK. Towards effective participation of the private health sector in Ghana's COVID-19 response. Pan Afr Med J 2020;35:47.

24. Gerrard A. A Coordinated Public-Private Sector Response in Liberia to COVID-19. Health Policy Plus 2020. Accessed 1 March 2021. Available from: http://www.healthpolicyplus. com/liberiaHFL.cfm

25. Impact for Health for WHO. Engaging the private health sector in the COVID-19 response. Impact for Health; 2020. Accessed 1 March 2021. Available from: https://www.impactforhealth. com/post/engaging-the-private-health-sector-in-the-covid-19response 
